\title{
Productivismo y abandono: dos caras de la transición forestal en Galicia (España), 1966-2009
}

\author{
Productivism and abandonment: \\ the two sides of forest transition in Galicia (Spain), 1966-2009
}

\author{
Eduardo José Corbelle Rico ${ }^{\text {a*, José María Tubío Sánchez a }}$ \\ *Autor de correspondencia: ${ }^{\text {a }}$ Universidade de Santiago de Compostela, Escuela Politécnica Superior de Ingeniería, \\ Departamento de Ingeniería Agroforestal, Lugo, España, tel: 34-982-823-324, eduardo.corbelle@usc.es
}

\begin{abstract}
SUMMARY
The expansion of wooded area is a common trait of most industrialized countries, and in every one of them it implies a trend reversal from the deforestation of previous centuries. The concept of "forest transition" was proposed in the last years of the twentieth century as a suitable designation for this process, for which several different explanations, or transition paths, were developed. Each transition path would likely result in a different species composition of the newly formed forest stands, and it is possible that several different paths could simultaneously operate in the same geographic area. In this work we present the case of Galicia, a Spanish region that has recently become the biggest producer of wood in the country, representing about one half of total production. We explore the trends followed by wooded areas using the four available editions of the Forest Map of Spain, from 1966 to 2009. Results allow identifying three different areas within the region according to the most common species in the newly formed forests. This division reveals the existence of a process of spatial specialization that concentrated the production of wood for the processing industry in a relatively small part of the whole territory, while leaving the rest of the region mostly void of active management. Results suggest that at least three different paths of forest transition (scarcity path, economic development path and State policy path) have been active in the region in the last fifty years, though not always in the same areas, neither with equal intensity along time.
\end{abstract}

Key words: reforestation, forest plantation, spontaneous revegetation, forest industry, eucalyptus.

\section{RESUMEN}

La expansión de la superficie arbolada es un fenómeno común a la mayoría de los países industrializados, y, en todos ellos, supone un cambio de tendencia respecto de la deforestación característica de siglos anteriores. A finales del siglo veinte se acuñó el concepto de «transición forestal» para referirse a este cambio, para el que se han propuesto diferentes explicaciones teóricas o caminos de transición. Diferentes caminos dan lugar a diferentes tipos de masa, y pueden actuar en localizaciones relativamente cercanas dentro de la misma área geográfica. En este trabajo se presenta el caso de Galicia (España), una región especializada en la producción de madera hasta el punto de representar la mitad de la producción del país, y se analiza la evolución de la superficie arbolada a través de las cuatro ediciones disponibles del Mapa Forestal de España, desde 1966 hasta 2009. Los resultados permiten identificar tres grandes zonas dentro de la región, diferenciadas entre sí por la composición dominante de las masas creadas. Esta división evidencia la existencia de un intenso proceso de especialización espacial que concentró la producción de madera de uso industrial en una parte relativamente reducida del territorio, dejando el resto sin apenas gestión ni aprovechamiento económico. Los resultados sugieren que al menos tres caminos diferentes de la transición forestal (escasez, desarrollo económico, y políticas de Estado) han actuado en la región en el último medio siglo, aunque no siempre sobre las mismas áreas simultáneamente ni con igual intensidad a lo largo del tiempo.

Palabras clave: reforestación, plantación forestal, revegetación espontánea, industria forestal, eucalipto.

\section{INTRODUCCIÓN}

En un contexto global de deforestación, la superficie total de bosque templado a nivel mundial está en aumento. En Europa este es un proceso observable desde, al menos, la década de 1950 (Fuchs et al. 2015), aunque en algunos casos haya comenzado antes: en España, por ejemplo, la superficie forestal arbolada total pasó de cerca de seis mi- llones de hectáreas a finales del siglo diecinueve hasta los dieciséis millones existentes en la actualidad (Vadell et al. 2016). Después de observar procesos similares en varios países, el geógrafo escocés A. Mather acuñó el término «transición forestal» para denominar a esta regularidad empírica: el paso de un período secular de deforestación neta a otro de reforestación neta en países del hemisferio norte (Mather y Needle 1998). 
Mather y Needle (1998) explicaron la expansión de la superficie arbolada como el resultado de la concentración de la producción agrícola en las tierras de mejor calidad. Un uso más intensivo de estas áreas habría permitido prescindir de zonas marginales para la producción de pasto o alimentos, y estas últimas quedarían libres para ser ocupadas por el arbolado. Rudel et al. (2005) denominaron a esta explicación el camino del desarrollo económico (economic development path) de la transición forestal, a la que añadieron una segunda: el camino de la escasez (scarcity path) de productos forestales. Este estaría relacionado con la reducción de la oferta de productos forestales (de la madera, por ejemplo) y, por lo tanto, con el incremento de los precios de estos productos que llevaría a una parte de los propietarios a realizar plantaciones en tierras hasta entonces utilizadas para otro tipo de propósitos.

Más tarde, otros autores han incorporado nuevas explicaciones para completar un marco teórico unificado de la transición forestal. Lambin y Meyfroidt (2010) añadieron tres caminos adicionales: el camino de la política de Estado (State policy), el de la intensificación productiva de los pequeños propietarios (smallholder intensification) y el de la globalización económica. El primero de ellos puede parecer similar al camino de la escasez, pero se diferencia de aquel en que las motivaciones que llevan a aumentar la superficie arbolada se sitúan en este caso fuera del sector forestal y persiguen otros objetivos como, por ejemplo, la creación de empleo o la promoción del turismo. El camino de la intensificación tiene relación con la expansión de plantaciones para producción de fruto, así como setos y otras estructuras de borde de parcela, o bien la implantación de sistemas agroforestales. Todos ellos elementos de los sistemas productivos, en definitiva, que demandan tiempo de trabajo y conocimientos adicionales (es decir, son más intensivos en mano de obra que otros, más convencionales) y que son parte común de las estrategias de diversificación productiva de muchas explotaciones de pequeño tamaño. Finalmente, el camino de la globalización se refiere a fenómenos de reforestación que tienen su origen en presiones originadas lejos de las fronteras nacionales, como acuerdos internacionales, presiones de grupos conservacionistas, o la adquisición de tierras (o derechos de uso) por empresas radicadas en terceros países. En ocasiones se ha atribuido a la transición forestal un efecto ambiental positivo, por ejemplo, en lo relativo a la captura y fijación de carbono o la protección del suelo frente a la erosión. En todo caso, los efectos concretos dependerán de la composición y localización de las masas creadas, así como del papel que los recursos forestales juegan en el conjunto del sistema socioecológico (Gingrich et al. 2016). Al no tener en cuenta estas variables, la formulación inicial de la teoría de transición forestal ha sido objeto de críticas que señalan la necesidad de integrar su estudio en el marco más amplio de los cambios de uso/cubierta del suelo (e.g. Perz 2007).

Galicia es una de las diecisiete comunidades autónomas que forman el territorio de España y está situada en el noroeste de la Península Ibérica (figura 1). Presenta un área total de $29.575 \mathrm{~km}^{2}$ y un clima oceánico mediterráneo con verano suave (Csb en la clasificación de KöppenGeiger). Se trata de un buen ejemplo de área periférica y ligeramente montañosa dentro del conjunto de Europa. El régimen de propiedad es mayoritariamente privado, bien a título individual o bien en forma de propiedad comunal. En paralelo con las transformaciones del sector agrícola y ganadero de las últimas décadas, la producción forestal ha ido ganando dentro de la producción total del Estado. Las estimaciones publicadas por las diferentes ediciones del Inventario Forestal Nacional (IFN) español indican que la superficie arbolada pasó de 1,1 millones de hectáreas en 1974 a 1,4 millones en 2009 (IFN 1 e IFN 4 respectivamente; la diferencia en escalas de trabajo y definiciones empleadas en cada uno puede matizar esta apreciación). Si bien este ritmo de aumento ( $27 \%$ en 35 años) es inferior a la media del conjunto de España, las especiales características biofísicas de esta parte del país la sitúan entre las áreas más productivas de Europa (Verkerk et al. 2015) y explican que el incremento de biomasa arbórea total haya sido, por el contrario, muy superior al global de España ( $272 \%$ en el mismo período). Con un área total que supone algo menos del $6 \%$ del total de España, Galicia produjo durante el último decenio entre seis y ocho millones de metros cúbicos con corteza anuales, que suponen alrededor del 50-60\% del volumen total de cortas del Estado, y alrededor del 60-70 \% de las cortas de eucalipto (INE 2017). La producción de tableros y de pasta de papel constituyen, conjuntamente, el destino mayoritario de la madera cortada.

En el año 1992 el gobierno autonómico aprobó el Plan Forestal de Galicia (PFG) con el objetivo de racionalizar e impulsar la producción forestal, a través del estímulo de la inversión privada, la mejora de la organización de la Administración forestal y «la erradicación de los incendios forestales» (Xunta de Galicia 1992). El PFG se elaboró con un horizonte temporal de 40 años (hasta 2032), al final del cual el volumen de cortas debería llegar a los 14 millones de metros cúbicos anuales, de los cuales siete millones de coníferas, cinco millones de eucalipto y dos millones de frondosas caducifolias. En 2014, el Consello Forestal de Galicia, un órgano consultivo y asesor de la Administración forestal en el que participan diferentes actores de la Administración y la sociedad civil, acordó crear un grupo de trabajo para iniciar el proceso de revisión del PFG. El grupo de trabajo dio por finalizadas sus actividades en 2015 y aprobó un documento de directrices para la redacción de una revisión del plan (Xunta de Galicia 2015) que abordan fundamentalmente la percepción de los actores sociales sobre el grado de cumplimiento del plan anterior y las líneas de actuación preferentes para su revisión. El documento de directrices es claro al referir que la percepción del grado de cumplimiento por parte de los diferentes actores es, en general, muy baja. La única evaluación cuantitativa y cualitativa del grado de cumplimiento de los 
principales objetivos del PFG de la que se tiene constancia es la que figura en el Documento Previo de revisión (Xunta de Galicia 2016), pero esta apenas profundiza en la evolución seguida por las áreas ocupadas por los diferentes tipos de masa y su comparación con las previsiones del plan.

El objetivo principal de este trabajo es estudiar la expansión de las masas arboladas en Galicia desde el punto de vista de la teoría de la transición forestal, con especial atención no sólo al tipo de masas creadas sino también a los posibles procesos de especialización espacial que pudieran tener lugar y a su cronología. El período de estudio comprende las cuatro ediciones del Mapa Forestal de España, desde 1966 hasta 2009. Comparar la evolución seguida por las masas arboladas con los objetivos del Plan Forestal de Galicia aprobado en 1992 y, por ende, la influencia de las políticas públicas recientes constituye un objetivo secundario que es tratado en este trabajo de modo preliminar y pendiente de mayor atención en análisis posteriores.

\section{MÉTODOS}

Materiales. La principal fuente de información de este trabajo está formada por las cuatro ediciones del Mapa
Forestal de España (MFE), publicadas a escala 1:400.000 (MFE400), 1:200.000 (MFE200), 1:50.000 (MFE50) y 1:25.000 (MFE25). Para el caso de Galicia, las fechas de referencia de cada edición son, respectivamente, 1966, 1989, 1999 y 2009 (las tres últimas ediciones están asociadas a la realización de la segunda a cuarta edición del IFN). Todas ellas fueron obtenidas en formato vectorial desde los servidores del Banco de Datos de la Naturaleza del Ministerio de Medio Ambiente de España. Los cambios en la escala de publicación de cada edición del mapa son consecuencia de los avances en la metodología de producción de cada edición, e inevitablemente suponen un problema para la interpretación de los resultados de este trabajo. Por ejemplo, el MFE400 fue preparado a partir de la información cartográfica sobre deslindes existente en los servicios forestales de la época. El tamaño de la unidad mínima cartografiable se sitúa en 20-30 hectáreas. El MFE200 se apoyó sobre fotografía aérea analógica en blanco y negro. El MFE50 fue realizado a partir de imágenes de base tomadas por el satélite IRS-1. El tamaño de la unidad mínima cartografiable es de 2,5 ha para teselas con arbolado y 6,25 ha para teselas desarboladas. Finalmente, el MFE25 se realizó a partir de ortofotografías aéreas asociadas al Plan Nacio-
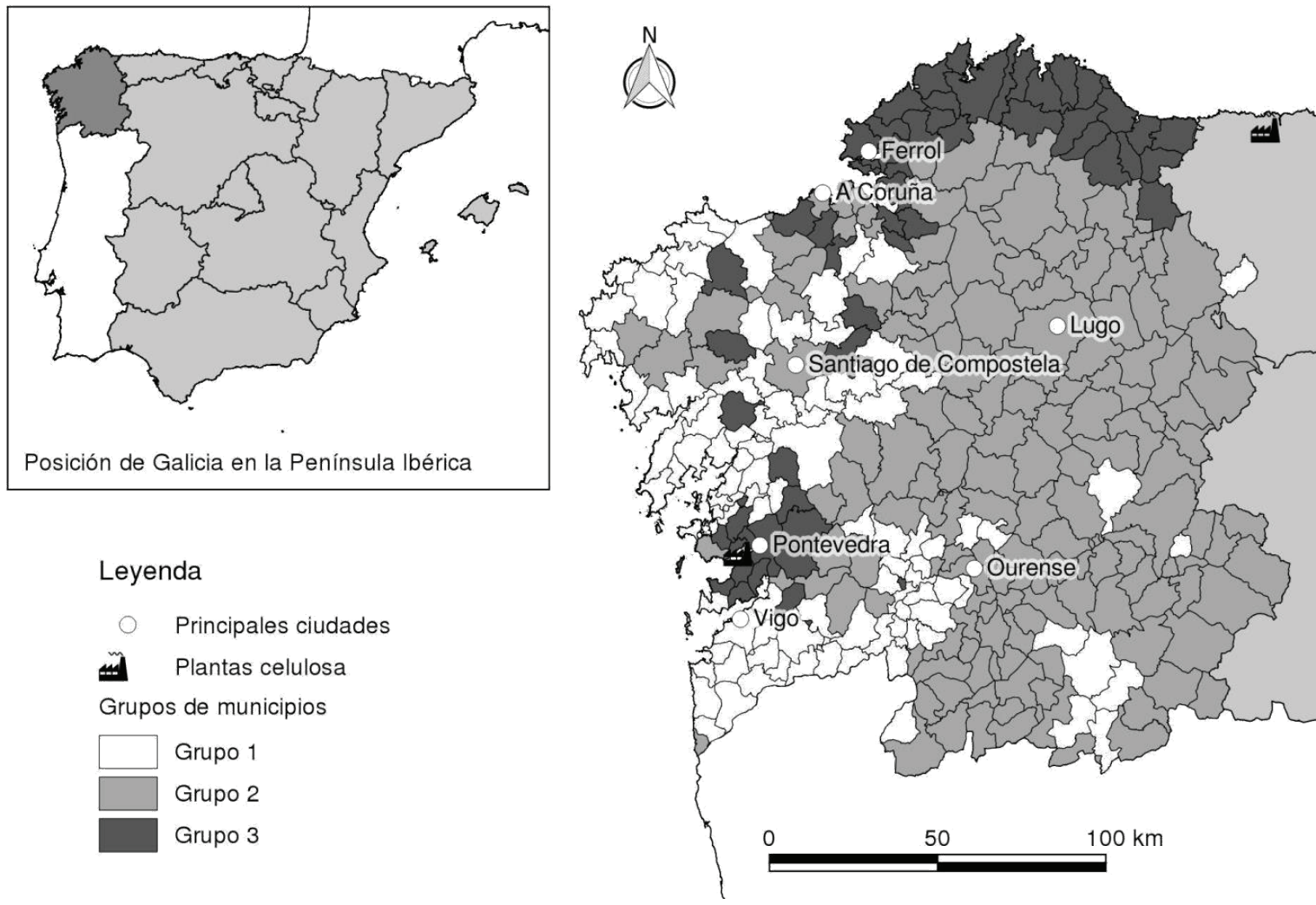

Figura 1. Localización del área de estudio y división en grupos de municipios resultante del análisis de conglomerados. Para facilitar la interpretación se ha incluido la situación de las principales ciudades y de las plantas de pasta de papel de Lourizán (Pontevedra) y Navia (Asturias).

Location of the study area and groups of municipalities as resulting from the cluster analysis. For illustration purposes, the map indicates the location of main cities and pulpwood plants of Lourizán (Pontevedra) and Navia (Asturias). 
nal de Ortofotografía Aérea (PNOA), con resolución espacial de 0,5-1 m/píxel. El tamaño de la tesela mínima de arbolado es de 1 ha (1-2 ha para las teselas desarboladas).

También se emplearon algunas otras fuentes de carácter estadístico como el Censo de Población y Viviendas o el Censo Agrario, ambos producidos por el Instituto Nacional de Estadística (INE), así como diferentes fuentes estadísticas sobre cortas de madera: Estadística Forestal de España (1960-1971), Anuario de Estadística Agraria (1972-2004), Anuario de Estadística Forestal (2005-2011).

Análisis. Para permitir la comparación entre diferentes ediciones del MFE fue necesario realizar previamente una homogeneización temática y geométrica. Para la primera de ellas se redujo la leyenda a seis grandes clases de formación arbolada: coníferas, eucalipto (fundamentalmente Eucalyptus globulus Labill.), otras frondosas diferentes del eucalipto, y las posibles masas mezcladas entre las tres categorías anteriores. Cada una de las formaciones identificadas en los mapas originales fue reclasificada en una de las seis categorías objeto de estudio. En el caso del MFE400 y MFE200 no se emplearon clases de masas mezcladas, dado que las clases originales no presentan suficiente precisión temática y clasifican las masas únicamente en función de la especie principal (con la única excepción de las formaciones «Bosque mixto de caducifolios y coníferas» $\mathrm{y}$ «Mezcla de coníferas y frondosas con predominio de exóticas», presentes en el MFE200, que fueron reclasificadas como mezcla de coníferas y otras frondosas). En el caso del MFE50 y MFE25 sí existía detalle suficiente para identificar más formaciones como mezcla de alguna de las categorías principales. En la mayoría de los casos no se trata de masas mixtas en sentido estricto sino más bien de pequeñas manchas de masas puras de distintas especies que fueron registradas conjuntamente al ser su tamaño individual inferior al de la unidad mínima cartografiable. Para conseguir cierta homogeneidad geométrica, todos los mapas fueron convertidos a espacio ráster con una resolución de $25 \mathrm{~m} /$ píxel.

Para detectar posibles procesos de especialización espacial se realizó un análisis de conglomerados sobre la proporción de superficie total municipal (314 municipios) correspondiente a cada una de las seis clases de formación arbolada, en cada año. Se empleó el método de agrupamiento jerárquico de Ward sobre la distancia euclídea entre observaciones; pero no se utilizó ningún procedimiento de estandarización previa de las variables, dado que todas se encontraban dentro del rango 0-1. Se determinó el número de grupos a formar sobre el dendrograma resultante del agrupamiento, y se utilizaron diagramas de cajas para interpretar su significado. Posteriormente se comparó el comportamiento de los grupos de municipios resultantes con algunas variables auxiliares como la altitud media del municipio, la evolución de su población, o la evolución del número de explotaciones.

Finalmente, se analizaron los objetivos de superficie ocupada por diferentes especies conforme fue propuesto en el Plan Forestal de Galicia de 1992 y se compararon con la evolución seguida desde el MFE200 de 1989 hasta el MFE25 de 2009. Para realizar la comparación fue necesario recurrir a las categorías originales de cada uno de los mapas, y no a la leyenda homogénea de seis clases utilizada para los cálculos ya mencionados.

\section{RESULTADOS}

Área y composición de las masas arboladas. Los resultados confirman el aumento sostenido de la superficie arbolada a lo largo de todo el período estudiado: 974.289 ha en 1966 (33,0\% de la superficie total de Galicia), 1.060 .893 ha en 1989 (35,9\%), 1.359.817 ha en 1999 (45,9\%), 1.416 .094 ha en 2009 (47,9\%). El ritmo de aumento se hizo más acusado en el período 1989-1999, con una expansión media de aproximadamente 30.000 ha por año, en comparación con los períodos anterior y posterior (alrededor de 3.800 y 5.900 ha por año, respectivamente). La expansión de la superficie ocupada fue acompañada por un cambio en la composición de las masas existentes. A lo largo de todo el período la superficie ocupada por masas de coníferas disminuyó, desde 772.825 ha en 1966 a 399.413 ha en 2009 (excluidas las masas mezcladas con eucalipto $\mathrm{u}$ otras frondosas). El principal descenso de la superficie de coníferas se produjo entre 1966 y 1999, a un ritmo aproximado de 10.000 ha anuales. Entre 1989 y 1999 continuó la misma tendencia, pero a un ritmo inferior de aproximadamente $3.000 \mathrm{ha} / \mathrm{año}$. Por el contrario, la superficie ocupada por masas de eucalipto pasó de 14.235 ha en 1966 a 285.714 ha en 2009, con un ritmo más intenso al principio del período estudiado (alrededor de 6.700 ha/año en 1966-1989, 6.000 ha por año en $1989-1999$ y 5.000 ha por año en 1999-2009). La superficie ocupada por otras especies frondosas también aumentó, desde 187.229 ha en 1966 a 423.262 ha en 2009, también con un ritmo progresivamente más lento (7.000 ha por año en 1966-1989, 6.000 ha por año en 1989-1999 y 1.500 ha por año en 1999-2009). Por otra parte, en los datos correspondientes a 1999 y 2009 (MFE50 y MFE25) es posible identificar masas mezcladas que no aparecen en los años anteriores. Este es el caso de las masas de coníferas mezcladas con eucalipto (182.827 ha en 1999), las de coníferas con otras frondosas (82.550 ha), y las de eucalipto con otras frondosas (21.654 ha). Mientras la primera de ellas apenas varió en 2009, las dos últimas experimentaron un aumento de 12.699 y 7.360 ha, respectivamente.

Se pueden observar los cambios entre categorías a lo largo de los años en los cuadros 1,2 y 3 y una versión simplificada en la figura 2A. En ellas se puede apreciar cómo las nuevas masas de eucalipto fueron apareciendo en parte sobre superficies antes ocupadas por coníferas y en parte sobre superficies sin formación arbolada previa: 71.329 ha y 84.773 ha, respectivamente, en 1966-1989; 52.308 ha y 72.531 ha en $1989-1999 ; 21.235$ ha y 51.450 ha en 1999-2009. Las masas mezcladas de coníferas con eucalipto funcionaron en gran medida como un paso intermedio en la conversión de masas puras de coníferas en masas puras de eucalipto: de las 182.827 ha consignadas 
Cuadro 1. Matriz de cambios, Galicia, años 1966-1989 (ha). Los datos en filas corresponden a las superficies en 1966, los datos en columnas a 1989. (Leyenda: SFA - sin formación arbolada, C - coníferas, E - eucalipto, OF - Otras frondosas).

Transition matrix, Galicia, 1966-1989 (ha). Data in rows correspond to 1966, data in columns to 1989 (Legend: SFA - area without forest cover, C - conifers, E - eucalyptus, OF - hardwood species other than eucalyptus).

\begin{tabular}{lrrrrrr}
\hline \multirow{2}{*}{ Año 1966 } & \multicolumn{7}{c}{ Año 1989} \\
\cline { 2 - 7 } & \multicolumn{1}{c}{ C } & C-OF & \multicolumn{1}{c}{ E } & \multicolumn{1}{c}{ OF } & \multicolumn{1}{c}{ SFA } & \multicolumn{1}{c}{ Total } \\
\hline C & 268.089 & 91 & 71.329 & 45.546 & 387.770 & 772.825 \\
E & 1.500 & 1 & 6.449 & 228 & 6.058 & 14.235 \\
OF & 14.556 & 0 & 7.027 & 55.241 & 110.405 & 187.229 \\
SFA & 258.157 & 127 & 84.773 & 247.779 & 1.391 .873 & 1.982 .708 \\
\hline Total & 542.301 & 219 & 169.578 & 348.795 & 1.896 .106 & 2.956 .998 \\
\hline
\end{tabular}

Cuadro 2. Matriz de cambios, Galicia, años 1989-1999 (ha). Los datos en filas corresponden a las superficies en 1989, los datos en columnas a 1999. (Leyenda: SFA - sin formación arbolada, C - coníferas, E - eucalipto, OF - Otras frondosas).

Transition matrix, Galicia, 1989-1999 (ha). Data in rows correspond to 1989, data in columns to 1999 (Legend: SFA - area without forest cover, C - conifers, E - eucalyptus, OF - hardwood species other than eucalyptus).

\begin{tabular}{lrrrrrrrr}
\hline \multirow{2}{*}{ Año 1989 } & \multicolumn{7}{c}{ Año 1999} \\
\cline { 2 - 8 } & \multicolumn{1}{c}{ C } & \multicolumn{1}{c}{ C-E } & \multicolumn{1}{c}{ C-OF } & \multicolumn{1}{c}{ E } & \multicolumn{1}{c}{ E-OF } & \multicolumn{1}{c}{ OF } & \multicolumn{1}{c}{ SFA } & \multicolumn{1}{c}{ Total } \\
\hline C & 245.287 & 87.413 & 21.563 & 52.308 & 3.899 & 26.365 & 105.467 & 542.301 \\
C-OF & 4 & 0 & 0 & 130 & 0 & 43 & 42 & 219 \\
E & 9.752 & 25.016 & 700 & 99.770 & 4.558 & 3.233 & 26.549 & 169.578 \\
OF & 26.332 & 11.151 & 19.357 & 7.481 & 4.475 & 172.156 & 107.842 & 348.795 \\
SFA & 150.953 & 59.247 & 40.930 & 72.531 & 8.723 & 206.441 & 1.357 .281 & 1.896 .106 \\
\hline Total & 432.328 & 182.827 & 82.550 & 232.220 & 21.654 & 408.238 & 1.597 .180 & 2.956 .998 \\
\hline
\end{tabular}

Cuadro 3. Matriz de cambios, Galicia, años 1999-2009 (ha). Los datos en filas corresponden a las superficies en 1999, los datos en columnas a 2009. (Leyenda: SFA - sin formación arbolada, C - coníferas, E - eucalipto, OF - Otras frondosas).

Transition matrix, Galicia, 1999-2009 (ha). Data in rows correspond to 1999, data in columns to 2009 (Legend: SFA - area without forest cover, C - conifers, E - eucalyptus, OF - hardwood species other than eucalyptus).

\begin{tabular}{|c|c|c|c|c|c|c|c|c|}
\hline \multirow{2}{*}{ Año 2009} & \multicolumn{8}{|c|}{ Año 1999} \\
\hline & $\mathrm{C}$ & C-E & $\mathrm{C}-\mathrm{OF}$ & E & E-OF & $\mathrm{OF}$ & SFA & Total \\
\hline $\mathrm{C}$ & 227.373 & 43.210 & 28.461 & 21.235 & 3.515 & 25.762 & 82.773 & 432.328 \\
\hline C-E & 28.928 & 58.750 & 4.844 & 45.027 & 4.416 & 9.292 & 31.569 & 182.827 \\
\hline $\mathrm{C}-\mathrm{OF}$ & 12.850 & 4.428 & 22.215 & 1.576 & 1.069 & 20.975 & 19.438 & 82.550 \\
\hline E & 8.338 & 24.229 & 1.780 & 152.654 & 6.430 & 6.552 & 32.238 & 232.220 \\
\hline E-OF & 718 & 1.677 & 562 & 6.916 & 3.581 & 4.953 & 3.247 & 21.654 \\
\hline $\mathrm{OF}$ & 19.381 & 9.832 & 16.757 & 6.856 & 3.955 & 245.763 & 105.695 & 408.238 \\
\hline SFA & 101.825 & 41.317 & 20.630 & 51.450 & 6.050 & 109.966 & 1.265 .942 & 1.597 .180 \\
\hline Total & 399.413 & 183.442 & 95.249 & 285.714 & 29.014 & 423.262 & 1.540 .904 & 2.956 .998 \\
\hline
\end{tabular}


en 1999, 87.413 ha ocuparon áreas anteriormente ocupadas por masas puras de coníferas y sólo 25.016 ha ocuparon áreas anteriormente pobladas con eucalipto. Aunque el área total de esta categoría se mantuvo prácticamente estable hasta 2009 (183.442 ha), a lo largo de los años es evidente la transición hacia las masas puras de eucalipto: entre 1999 y 2009, solo 28.928 ha de masas mezcladas de este tipo se convirtieron en masas puras de coníferas, pero 45.027 ha pasaron a ser masas puras de eucalipto. Por el contrario, la expansión de las masas de otras frondosas distintas del eucalipto se produjo fundamentalmente sobre terrenos anteriormente desarbolados $\mathrm{y}$, en mucha menor medida, sobre terrenos anteriormente poblados por coníferas.

Procesos de especialización espacial. Los resultados del análisis de conglomerados permitieron identificar tres grandes grupos de municipios (figura 2). Cada grupo define un área geográfica dentro de la comunidad autónoma que muestra una evolución apreciablemente distinta de la superficie arbolada total y, sobre todo, de su composición. Los municipios incluidos en los grupos 1, 2 y 3 suman, respectivamente, el 25, 60 y $15 \%$ de la superficie de la comunidad. En el mapa se puede apreciar claramente cómo los grupos 1 y 3 se corresponden con municipios del área costera, situados por debajo de los 44 metros de altitud sobre el nivel del mar, mientras que el grupo 2 incluye fundamentalmente municipios del interior. Los municipios incluidos en el grupo 1 se caracterizan por haber pasado del dominio de las masas de coníferas (alrededor del $48 \%$ de la superficie total en 1966) a una situación donde las coníferas todavía ocupan la mayor parte de la superficie arbolada ( $22 \%$ de la superficie total en 2009) pero coexisten con masas mezcladas de coníferas con eucalipto $(10 \%)$ y masas puras de eucalipto (9\%). Los municipios del grupo 3 también mostraban en 1966 un claro dominio de las masas de coníferas ( $37 \%$ de la superficie total en 1966), pero hacia 1989 ya es evidente una transición hacia un modelo de masa dominado por el eucalipto (29\%) que se iría con-
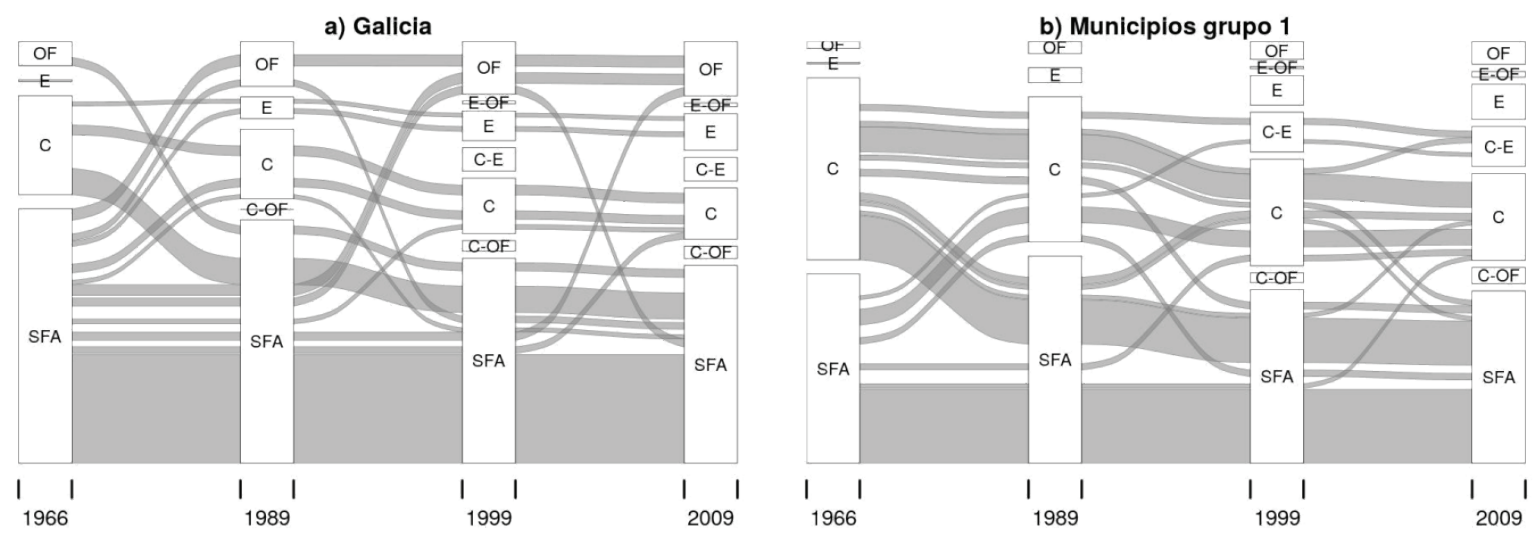

c) Municipios grupo 2
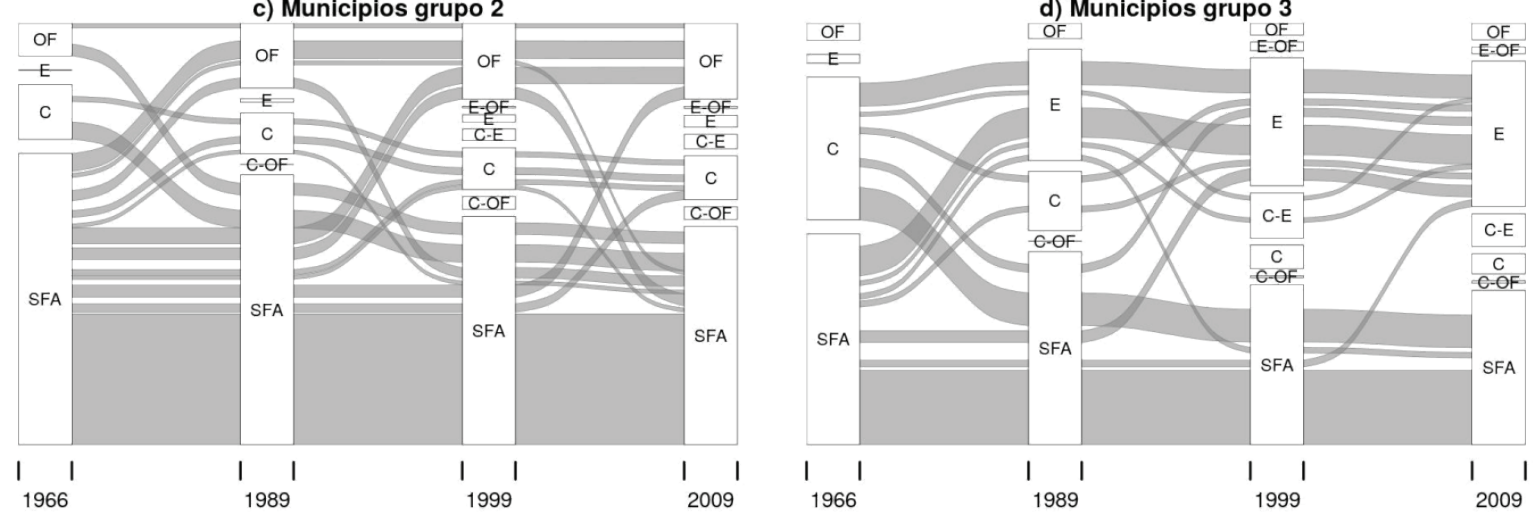

Figura 2. Diagramas aluviales de cambios entre superficies. Las barras verticales (en blanco) representan la distribución de superficie entre las diferentes categorías en cada año analizado. Las barras de flujo (en gris) representan flujos de superficie entre categorías a lo largo del tiempo y su ancho es proporcional a la superficie que representan. Para facilitar la lectura se han excluido los flujos que no superan el $1 \%$ de la superficie total de Galicia (A) o cada grupo de municipios (B, C, D). (Leyenda: SFA - sin formación arbolada, $\mathrm{C}$ - coníferas, E - eucalipto, OF - Otras frondosas.).

Alluvial diagrams showing changes along time. Vertical bars (in white) represent the distribution of area among different categories of land cover in each year. Flow bars (in grey) represent changes among categories along time, their width being proportional to the amount of area they represent. For the sake of simplicity, flows representing less than $1 \%$ of total area of Galicia (A) or the corresponding group of municipalities (B, C, D) were omitted (Legend: SFA - area without forest cover, C - conifers, E - eucalyptus, OF - hardwood species other than eucalyptus). 
solidando con el tiempo. Las masas puras de eucalipto en el grupo 3 suponían el $38 \%$ de la superficie total en 2009 . En cualquiera de los dos grupos mencionados la presencia de masas de otras frondosas fue siempre residual, en ningún caso superior al $6 \%$ de la superficie total.

En comparación con lo descrito para los grupos 1 y 3, los municipios englobados en el grupo 2 muestran una evolución muy diferente. La situación de 1966 muestra la coexistencia de masas de coníferas y especies frondosas distintas del eucalipto, con una leve diferencia a favor de las primeras (14\% frente a $8 \%$ de la superficie total), pero con el paso del tiempo esta situación se va decantando a favor de las masas de otras frondosas, hasta ocupar el $20 \%$ de la superficie total del grupo en 2009. La presencia de eucalipto es muy reducida en la mayor parte de los municipios de este grupo y no suponía en 2009 más que el $3 \%$ de la superficie total.

La población total de los municipios incluidos en los grupos 1 y 3 muestra una tendencia sostenida de aumento a lo largo del período estudiado, mientras que la de los incluidos en el grupo 2 decrece de manera sostenida. Por el contrario, al analizar los datos del número de explotaciones registradas en los Censos Agrarios de 1962, 1982, 1989,1999 y 2009, no se pudo observar diferencias significativas en la evolución seguida por los diferentes grupos.
Los cambios entre formaciones arboladas en cada grupo (figura 2B, C y D) reproducen, en general, los resultados para el conjunto del área de estudio, pero con ciertos matices de relevancia. En primer lugar, destaca el hecho de que la superficie arbolada total en el conjunto del grupo 1 descendió durante el período 1966-1989, en contra de la tendencia general, debido a la reducción del área ocupada por masas de coníferas y su transformación en áreas desarboladas. En los tres grupos, además, el crecimiento de la superficie arbolada es mayor en el período 1989-1999 que en los otros dos, pero este efecto es mucho mayor en el grupo 2: de hecho, de las alrededor de 300.000 ha en que se incrementa la superficie arbolada en ese período en toda Galicia, casi dos tercios están situadas en el grupo 2. En términos de balance neto, la superficie incrementada en el grupo 2 en 1989-1999 se reparte a partes casi iguales entre masas mixtas de coníferas y eucalipto, masas mixtas de coníferas $\mathrm{y}$ frondosas, y masas de frondosas diferentes del eucalipto.

Comparación con los objetivos del Plan Forestal de 1992. Para comparar los cambios observados en las formaciones arboladas existentes con los objetivos del Plan Forestal de Galicia (PFG) aprobado en 1992, se presenta en el cuadro 4 la situación de partida antes de su aprobación

Cuadro 4. Comparación entre las superficies estimadas en diferentes ediciones del Mapa Forestal y las previsiones del Plan Forestal de Galicia (aprobado en 1992) para el año 2032. Todas las cifras en hectáreas.

Comparison of forest areas as estimated by the Forest Plan of Galicia and the data included in the Forest Map of Spain in 1989, 1999 and 2009 (data in hectars).

\begin{tabular}{|c|c|c|c|c|c|}
\hline Categoría & MFE400 (1966) & MFE200 (1989) & MFE50 (1999) & MFE25 (2009) & PFG (2032) \\
\hline Pinus pinaster Aiton & 675.391 & 456.993 & 331.605 & 264.566 & 334.132 \\
\hline Pinus radiata D. Don. & 13.082 & 32.632 & 55.209 & 87.423 & 231.207 \\
\hline Pinus sylvestris L. & 84.598 & 42.677 & 37.372 & 38.422 & 76.441 \\
\hline Pinus uncinate Ram. & --- & -- & -- & 5 & 133 \\
\hline Pinus nigra Arn. & --- & -- & 113 & 20 & 22.396 \\
\hline Pseudotsuga menziesii (Mirb.) Franco & --- & -- & 651 & 1.114 & 70.234 \\
\hline Otras coníferas & 17 & 7.698 & 23.788 & 9.048 & -- \\
\hline Total coníferas & 773.088 & 540.000 & 448.738 & 400.598 & 734.543 \\
\hline Eucalyptus spp. & 14.250 & 169.122 & 230.176 & 283.170 & 245.654 \\
\hline Castanea sativa Mill. & 47.613 & 27.818 & 33.932 & 26.816 & 218.953 \\
\hline Otras frondosas & 139.529 & 320.273 & 383.911 & 396.433 & 189.814 \\
\hline Total otras frondosas & 187.142 & 348.091 & 417.843 & 423.249 & 408.767 \\
\hline M.m. coníferas y frondosas * & --- & 219 & 101.470 & 110.634 & -- \\
\hline M.m. coníferas y eucalipto & --- & -- & 168.891 & 168.060 & -- \\
\hline M.m. eucalipto y otras frondosas & --- & -- & 21.649 & 29.008 & -- \\
\hline Frondosas alóctonas invasoras & --- & 481 & 2.130 & 2.571 & -- \\
\hline Total superficie arbolada & 974.480 & 1.057 .913 & 1.390 .897 & 1.417 .290 & 1.388 .964 \\
\hline
\end{tabular}

* M.m.: masas mezcladas. 
(correspondiente con los MFE400 de 1966 y MFE200 de 1989), los datos de las siguientes ediciones del MFE y los objetivos de área para cada especie. Conviene recordar que los objetivos previstos se referían en el PFG a un horizonte temporal de cuarenta años, es decir, al año 2032. Observando los resultados de las grandes clases de formación arbolada, se puede apreciar fácilmente que tanto los objetivos para las masas de eucalipto como para otras frondosas se habrían cumplido sobradamente: los valores observados en 2009 son superiores a los objetivos planteados para 2032. Por el contrario, el objetivo para las masas de coníferas estaría muy lejos de conseguirse: en lugar de ir aumentando desde la superficie existente en 1989, el resultado ha sido el contrario.

Al analizar las principales especies de modo individual la imagen de éxito relativo que podría desprenderse del párrafo anterior se vuelve menos clara. Prácticamente ninguna de las principales especies contempladas en el plan está cerca de conseguir los objetivos planteados. Las únicas excepciones son las diferentes especies de eucalipto, que habrían superado en 2009 la superficie objetivo para 2032. Conviene, en todo caso, recordar la existencia de masas identificadas como masas mixtas en los mapas originales, cuya superficie podría ser atribuida, al menos en parte, a alguna de las especies propuestas en el plan.

\section{DISCUSIÓN}

Los resultados presentados evidencian que la superficie arbolada de Galicia creció durante todo el período de estudio pero no permiten poner fecha a la transición forestal propiamente dicha, que habría tenido lugar en una fecha más temprana. Trabajos publicados para el caso de España (Vadell et al. 2016, Cervera et al. 2019) y Portugal (Oliveira et al. 2017) sugieren que la transición forestal habría comenzado a finales del siglo diecinueve o principios del veinte, con un significativo aumento de ritmo a partir de la década de 1960 como consecuencia de la intensa transformación del sistema agrario preexistente. En cualquier caso, el concepto de transición forestal es útil como marco teórico de este trabajo en la medida en que permite proponer una explicación a la evolución de las masas arboladas, mediante el análisis de los posibles «caminos» seguidos. Parece razonable suponer que durante el período estudiado en este trabajo hayan intervenido al menos tres de los caminos propuestos en la bibliografía, aunque estos hayan sido más activos en diferentes áreas y momentos del tiempo. Por ejemplo, el camino de la escasez de productos forestales permitiría explicar, en lo fundamental, la expansión del arbolado en el área litoral la región, en la que la producción de madera para satisfacer las demandas de la industria habría sido el principal motor de cambio y las masas resultantes están dominadas por las principales especies de producción de madera de uso industrial. Por el contrario, sería el camino del desarrollo económico, asociado al abandono de las actividades agrarias y a procesos de revegetación espontánea, el que mejor permite explicar lo sucedido en el interior, donde las masas de nueva aparición están compuestas fundamentalmente por especies que en la actualidad apenas tienen uso industrial: las especies diferentes de coníferas o eucaliptos representan alrededor del $3 \%$ de las cortas totales (INE 2017) a pesar de ocupar al menos el $30 \%$ de la superficie arbolada y suponer el $26 \%$ de las existencias totales de madera en pie (Dirección General de Medio Natural y Política Forestal 2011).

Superpuesto sobre los dos caminos citados, que actuarían en mayor o menor medida a lo largo de todo el período de estudio, se podría situar el camino de las políticas de Estado, asociado en este caso a las políticas europeas de reestructuración del sector agrario y cuya influencia es claramente apreciable en el período 1989-1999. En ellas se incluían diferentes medidas orientadas a reducir el número de explotaciones agrarias y, por ende, la producción final del sector. Las asociadas al Reglamento europeo 2080/1992, en la forma de ayudas directas para la reforestación de tierras agrícolas, actuaron directamente sobre la superficie forestal fomentando la creación de plantaciones que, en lo fundamental, habrían tenido una finalidad de producción de madera (García Arias y Pérez Fra 2001). Otras, como los incentivos para el barbecho voluntario y la reducción de la carga ganadera, o para el retiro anticipado de los agricultores, actuaron de manera indirecta al liberar tierras que anteriormente estaban siendo utilizadas para la producción agrícola y ganadera. En su conjunto, estas políticas actuaron potenciando los dos principales procesos de reforestación que ya estaban en marcha en las diferentes áreas de Galicia.

En paralelo al aumento de la superficie arbolada, los resultados permiten apreciar la progresiva sustitución de la principal especie de producción de madera a mediados del siglo veinte (Pinus pinaster) por especies del género Eucalyptus (fundamentalmente E. globulus). Aproximadamente la mitad de las masas de eucalipto de nueva aparición en cada período estudiado aparece en localizaciones anteriormente ocupadas por masas de coníferas. Aunque el inicio de las plantaciones de eucalipto con finalidad comercial se sitúa en la década de 1940 (Prada Blanco 1991) su escasa presencia en los datos de 1966 evidencia que no habrían sido muy comunes antes de ese año. El período de expansión que se observa en los datos del MFE coincide con el cambio operado en la demanda por parte de la industria transformadora, en particular, por parte de la industria de la pasta de papel. Así, la planta de la Empresa Nacional de Celulosas (ENCE, actualmente Energía y Celulosas) situada en Pontevedra comenzó consumiendo exclusivamente madera de coníferas desde su implantación en 1962 (Rico Boquete 1997), pero fue adaptando progresivamente su proceso productivo para introducir cada vez más madera de eucalipto. Alrededor de 1989, este suponía más del $80 \%$ del volumen consumido (Prada Blanco 1991), y en la actualidad la planta se abastece por completo con madera de este tipo. La transformación del proceso 
productivo fue acompañada de un aumento de su capacidad de producción, desde las 30.000 Tm de pasta de 1962 a las $400.000 \mathrm{Tm}$ de la actualidad (a las que es necesario añadir $600.000 \mathrm{Tm}$ de la planta situada en Navia, Asturias, apenas unos kilómetros al este de la frontera con Galicia, creada alrededor de 1970), y por lo tanto del consumo. En total, el consumo actual de ambas plantas se sitúa alrededor de 4 millones de metros cúbicos de eucalipto por año (ENCE 2015ab), una cifra muy similar al volumen total de cortas (4,1 millones de metros cúbicos en 2011 y con tendencia creciente). La influencia de la demanda sobre la expansión del eucalipto es claramente apreciable en la distribución de los municipios del grupo 3, que se sitúan fundamentalmente en las proximidades de las plantas de pasta de papel (figura 1).

El aumento neto de la superficie arbolada fue acompañado de un aumento, también sostenido, del volumen de biomasa arbórea presente sobre el territorio. El volumen medio de biomasa arbórea por hectárea de superficie arbolada total habría pasado de $63 \mathrm{~m}^{3}$ en 1974 (IFN1) a $86 \mathrm{~m}^{3}$ en 1989 (IFN2), $97 \mathrm{~m}^{3}$ en 1999 (IFN3) y $135 \mathrm{~m}^{3}$ en 2009 (IFN4). No obstante, este proceso esconde la existencia de procesos de deforestación de cierta importancia que afectaron sobre todo a las masas de coníferas y de otras frondosas distintas del eucalipto, y que son patentes en los tres períodos de tiempo estudiados. Es evidente que una parte puede tratarse de superficies afectadas por incendios forestales, dado que la superficie quemada durante el período 1966-2009 fue de 1,79 millones de hectáreas, de las cuales al menos 718.023 ha correspondían a superficie arbolada (MAPA 2018). Pero otra parte de estas conversiones hacia superficie no arbolada podría ser atribuida a la conversión para uso agrícola, particularmente para la producción de forraje (Corbelle Rico y Crecente Maseda 2014).

Alrededor del año en que se inicia el período estudiado en este trabajo, la mayor parte de la superficie de Galicia estaba siendo utilizada, de modo más o menos intensivo, por la actividad agraria. Esto incluía a las tierras de monte, habitualmente utilizadas para el pastoreo de ganado o la producción de materia orgánica con la que fertilizar las tierras de uso más intensivo. Por lo tanto, la expansión del arbolado está íntimamente relacionada con los cambios sociales y tecnológicos que permitieron reducir la superficie utilizada por las explotaciones agrarias, así como el número de estas. Es decir, se trata de un cambio del papel que la superficie forestal juega en el sistema: de asegurar la fertilidad de los cultivos a la provisión de madera para uso industrial (Soto Fernández 2015). Sin embargo, los resultados no sugieren que el descenso del número de explotaciones haya sido significativamente distinto entre los grupos de municipios identificados en este trabajo. La diferencia entre los grupos no estribaría tanto en un mayor o menor descenso del número de explotaciones, sino más bien en el destino final de las superficies que dejaron de ser utilizadas: la plantación con finalidad de producción de madera en unos casos, el abandono y posterior reve- getación espontánea en otros. Al contrario, sí se pudo encontrar que las áreas donde la revegetación espontánea es el principal proceso de expansión de la superficie arbolada (las zonas del interior, menos aptas para el cultivo de E. globulus) muestran un descenso más acusado de la población total. En definitiva, la transición forestal en Galicia ha sido a la vez motor y resultado de la transformación del sistema agrario preexistente. La combinación de ambos procesos fue pareja con la industrialización de los sectores agroganadero y forestal y su especialización en la producción de leche y madera dentro del contexto español. Ya fue mencionada la importancia actual de Galicia en la producción de madera de España; en el caso de la leche de vaca se aprecia una evolución similar, de modo que la producción ha pasado de suponer el $18 \%$ del total español en 1960 al $38 \%$ en 2012 (Soto Fernández 2015). Dado que el papel del monte ya no es garantizar la fertilidad de las tierras de cultivo ni producir alimento adicional para el ganado, la intensificación de ambas producciones solo ha sido posible a través de la importación de alimento para el ganado desde el extranjero. Ahora bien, ¿cuál ha sido el papel de la política forestal reciente en el proceso descrito? El modelo de ocupación del suelo propuesto en el PFG de 1992 situaba la superficie forestal objetivo en dos millones de hectáreas. De ellas, aproximadamente 1,4 millones de hectáreas deberían corresponder a monte arbolado con función productora, a las que se sumarían 270.000 ha de vegetación espontánea (matorral) destinadas al uso cinegético o de pastoreo extensivo, y 340.000 ha de espacios naturales protegidos (Xunta de Galicia 1992). En términos generales, estas grandes magnitudes se acercan a lo previsto en el plan: la superficie de espacios naturales protegidos incluidos en la red europea Natura 2000 supone en la actualidad algo más de 350.000 ha (la comparación con el MFE25 indica que esta está esencialmente desarbolada: sólo el $23 \%$ cubierta por frondosas diferentes del eucalipto, el $10 \%$ cubierta por coníferas, y el $2 \%$ cubierta por eucalipto), mientras que la superficie forestal arbolada total no incluida en espacios protegidos supone alrededor de 1,28 millones de hectáreas. La apreciación es diferente si se analiza por especies. La comparación de los objetivos de superficie marcados en el PFG con la situación actual indica que, a excepción de la relativa al eucalipto, ninguna de las previsiones de área ocupada se cumplió hasta el momento. Es cierto que no es posible comparar las previsiones del plan (calculadas para el año 2032) con la situación en 2009 pero en todo caso, si se extrapolan las tendencias observadas hasta ese año, la conclusión fundamental se mantiene. El modelo de monte actualmente existente es bastante menos diverso que el propuesto en el Plan, la evolución seguida por las masas forestales desde el año 1966 no se vio afectada por su aprobación y continuó básicamente inalterada hasta la actualidad. A pesar de las declaraciones expresadas en el PFG respecto de la multifuncionalidad de los espacios forestales, la realidad parece haber continuado, en lo fundamental, las 
premisas productivistas que caracterizaron la actuación de la Administración durante buena parte del siglo veinte (Rico Boquete 1995). Soto Fernández (2015) resume la situación actual en la existencia de tres grandes modelos de utilización del espacio forestal: uno muy intensivo para la producción de madera de crecimiento rápido, ligado al eucalipto; un segundo, de menor intensidad y dominado por coníferas; y un tercer espacio de aprovechamiento casi nulo. Estos tres modelos son fácilmente asimilables a los tres grupos de municipios identificados en este trabajo. Es decir que, dentro del proceso de especialización regional de Galicia como principal proveedora de madera en España-que la llevó de producir el $22 \%$ del volumen de cortas de España en 1960 al 55-60 \% de la actualidad (70-80 \% de la madera de eucalipto)- se puede apreciar un nivel de especialización subregional que concentra la producción en el $40 \%$ de la superficie de la comunidad. Que en esta pequeña zona, que representa aproximadamente el 2,4 \% de la superficie del Estado, se concentren aproximadamente la mitad de las cortas de madera de España da una idea de hasta qué punto el modelo adoptado es intensivo, aun tratándose de una de las áreas de mayor productividad forestal de Europa (Verkerk et al. 2015).

Sin embargo, ciertos aspectos sugieren que el modelo basado en E. globulus está cerca del agotamiento: por un lado, esta especie ocupa en la actualidad la mayoría de la superficie arbolada presente en las áreas más aptas para su cultivo, lo que va dejando menos espacio disponible para continuar su expansión en el futuro. Por otro lado, incluso en esas áreas de mayor aptitud, los daños por hongos o insectos defoliadores se han multiplicado en los últimos años (Dirección General de Medio Natural y Política Forestal 2011). Como resultado, hace algunos años que la industria ha comenzado a impulsar el uso de especies alternativas (por ejemplo, Eucalyptus nitens (H. Deane et Maiden) Maiden) que, a pesar de tener menor rendimiento para la producción de pasta, presentan por el momento menos problemas sanitarios y además tienen mayor resistencia al frío -lo que amplía, extendiéndolas hacia el interior, las áreas aptas para su plantación.

Los resultados sugieren, por lo tanto, que existe una correspondencia razonable, en el espacio y en el tiempo, entre la evolución de las necesidades de la industria transformadora y la evolución de la extensión y composición de las masas arboladas. Este proceso de hiperespecialización productiva y espacial continuó básicamente inalterado después de la aprobación del Plan Forestal de 1992, a pesar de que este tenía como objetivo alcanzar un modelo de monte más diversificado y una producción más repartida en el territorio, en la que otras especies tendrían un papel relevante. En la actualidad, este modelo de especialización productiva continúa pujante y parece difícil de revertir. Por ejemplo, durante los años 2015 a 2017 la Administración autonómica ha hecho circular varios borradores de un decreto que limitaría la expansión de especies del género Eucalyptus en la mitad suroriental de Galicia, que estarían justificados por haberse superado ampliamente las previsiones de la planificación forestal respecto del área ocupada por este tipo de especies. Sin embargo, una apreciable respuesta mediática por parte de asociaciones de propietarios y organizaciones cercanas a la industria maderera ha resultado, hasta el momento, en que ninguno de estos borradores fuera finalmente aprobado. Otros instrumentos normativos que ponían limitaciones a las plantaciones de especies de crecimiento rápido, como las Áreas de Especial Interés Paisajístico (AEIP) asociadas al Catálogo de paisaje de Galicia (Decreto 119/2016, de 28 de julio), generaron controversias dentro de la propia Administración que influyeron en la superficie y el alcance finalmente propuestos para las AEIP. En todo caso, existen otros instrumentos legales en vigor que permiten limitar la extensión de la superficie arbolada cuando esta supone un riesgo desde el punto de vista de incendios (por ejemplo la Ley 3/2007 de prevención y defensa contra incendios forestales, posteriormente modificada por la Ley 7/2012 de Montes, que a su vez introdujo limitaciones adicionales), cuando afecta a superficie de cultivo agrícola (Ley 6/2011 de movilidad de tierras), o cuando afecta a espacios naturales protegidos (Plan Director de Red Natura 2000). La Administración local, por otra parte, tuvo en la Ley del Suelo de Galicia (Ley 9/2002) un poderoso instrumento para proteger las áreas de cultivo de mayor interés mediante la clasificación de suelo rústico de especial protección agropecuaria, pero la mayor parte de los municipios no aprobó las ordenanzas necesarias o simplemente no realiza ningún seguimiento de los cambios de uso ocurridos (Tubío Sánchez y Crecente Maseda 2016).

\section{CONCLUSIONES}

Los resultados de este trabajo reafirman la complejidad de los procesos de cambio de uso o cubierta del suelo y, en particular, de la transición forestal. Se demuestra cómo una fuerte especialización productiva dentro del sector forestal de España ha convivido con otros procesos de expansión de la cubierta arbolada asociados a la ausencia de gestión, y se resalta la importancia de las políticas de ámbito europeo en la aceleración de ambos. Al mismo tiempo, la conformación de las nuevas masas arboladas aparece fuertemente condicionada por las necesidades de la principal industria consumidora, en contra (o cuando menos al margen) de las previsiones y directrices de la planificación forestal establecida por el gobierno regional, lo que resultó en una especialización espacial dentro de la región.

\section{REFERENCIAS}

Cervera T, J Pino, J Marull, R Padró, E Tello. 2019. Understanding the long-term dynamics of forest transition: From deforestation to afforestation in a Mediterranean landscape (Catalonia, 1868-2005). Land Use Policy 80: 318-331. DOI: 10.1016/j.landusepol.2016.10.006 
Corbelle Rico E, R Crecente Maseda. 2014. Urbanización, forestación e abandono. Cambios recentes na paisaxe de Galicia, 1985-2005. Revista Galega de Economía 23(1): 35-52.

Dirección General de Medio Natural y Política Forestal. 2011. Cuarto Inventario Forestal Nacional. Galicia. Madrid, España. Ministerio de Medio Ambiente, Medio Rural y Marino. $49 \mathrm{p}$.

ENCE (Energía y Celulosa, S.A., ES). 2015a. Centro de Operaciones de Navia - CEASA. Declaración Ambiental 2015. Madrid, España. ENCE, Energía y Celulosa. 48 p.

ENCE (Energía y Celulosa, S.A., ES). 2015b. Centro de Operaciones de Pontevedra. Declaración Ambiental 2015. Madrid, España. ENCE, Energía y Celulosa. 42 p.

Fuchs R, M Herold, P H Verburg, J G Clevers, J Eberle. 2015. Gross changes in reconstructions of historic land cover/use for Europe between 1900 and 2010. Global Change Biology 21(1): 299-313. DOI: $10.1111 /$ gcb. 12714

García Arias A I, M Pérez Fra. 2001. Análisis y evolución de la aplicación en Galicia del programa de ayudas a la reforestación de tierras agrarias (Reg. CEE 2080/92). Revista Galega de Economía 10(1): 151-176.

Gingrich S, C Lauk, T Kastner, F Krausmann, H Haberl, K Erb 2016. A Forest Transition: Austrian Carbon Budgets 18302010. In Haberl H, M Fischer-Kowalski, F Krausmann, V Winiwarters eds. Social Ecology Society-Nature Relations across Time and Space. Berlin, Alemania. Springer. p. 417427.

INE (Instituto Nacional de Estadística, ES). 2017. Estadística Anual de Cortas de Madera, 2005-2013. Consultado 2 nov. 2018. Disponible en https://www.ine.es/dyngs/IOE/es/operacion.htm? numinv $=01029$

Lambin EF, P Meyfroidt. 2010. Land use transitions: Socioecological feedback versus socio-economic change. Land Use Policy 27(2): 108-118. DOI: 10.1016/j.landusepol.2009.09.003

Mather AS, CL Needle. 1998. The forest transition: a theoretical basis. Area 30(2): 117-124.

MAPA (Ministerio de Agricultura, Pesca y Alimentación, ES). 2018. Estadística General de Incendios Forestales. Ministerio de Agricultura, Pesca y Alimentación (España). Consultado 29 oct. 2018. Disponible en: http://www.mapa.gob.es/ es/desarrollo-rural/estadisticas/incendios_default.aspx

Oliveira T M, N Guiomar, F O Baptista, J M Pereira, J Claro. 2017. Is Portugal's forest transition going up in smoke? Land Use Policy 66: 214-226. DOI: 10.1016/j.landusepol.2017.04.046

Perz S G. 2007. Grand theory and context-specificity in the study of forest dynamics: forest transition theory and other directions. The Professional Geographer 59(1): 105-114.

Prada Blanco A. 1991. Montes e industria: o circuito da madeira en Galicia. Santiago de Compostela, España. Fundación Caixa Galicia. 302 p.

Rico Boquete E. 1995. Política forestal e repoboacións en Galicia, 1941-1971. Santiago de Compostela, España. Servizo de Publicacións e Intercambio Científico, Universidade de Santiago de Compostela. 202 p.

Rico Boquete E. 1997. La creación de Celulosas de Pontevedra y su influencia en el sector forestal de la provincia. Madrid, España. Fundación Empresa Pública. 159 p.

Rudel T K, O T Coomes, E Moran, F Achard, A Angelsen, J $\mathrm{Xu}, \mathrm{E}$ Lambin. 2005. Forest transitions: towards a global understanding of land use change. Global Environmental Change 15(1): 23-31.

Soto Fernández D. 2015. Del manejo multifuncional del territorio a la desarticulación productiva: cambios en los flujos de biomasa durante el proceso de industrialización de la agricultura gallega (1960-2012). Documento de Trabajo 1505. Sevilla, España. Sociedad Española de Historia Agraria. 60 p.

Tubío Sánchez J M, R Crecente Maseda. 2016. Forcing and avoiding change. Exploring change and continuity in local land-use planning in Galicia (Northwest of Spain) and The Netherlands. Land Use Policy 50: 74-82. DOI: 10.1016/j. landusepol.2015.09.006

Vadell E, S de-Miguel, J Pemán. 2016. Large-scale reforestation and afforestation policy in Spain: A historical review of its underlying ecological, socioeconomic and political dynamics. Land Use Policy 55: 37-48. DOI: 10.1016/j.landusepol.2016.03.017

Verkerk P J, C Levers, T Kuemmerle, M Lindner, R Valbuena, P H Verburg, S Zudin. 2015. Mapping wood production in European forests. Forest Ecology and Management 357: 228-238. DOI: 10.5061/dryad.mk067/1

Xunta de Galicia. 1992. Plan Forestal de Galicia. Documento de síntesis. Santiago de Compostela, España. Dirección General de Montes y Medio Ambiente Natural. Consellería de Agricultura, Ganadería y Montes. 142 p.

Xunta de Galicia. 2015. Proposta de directrices para a revisión do Plan Forestal de Galicia. Documento final. Santiago de Compostela, España. Dirección Xeral de Ordenación e Produción Forestal. Consellería do Medio Rural. 112 p.

Xunta de Galicia. 2016. Primeira revisión do Plan Forestal de Galicia. Documento previo. Santiago de Compostela, España. Dirección Xeral de Ordenación e Produción Forestal. Consellería do Medio Rural. 84 p. 
